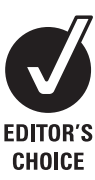

CHOICE

\title{
Obesity and risk of incident psoriatic arthritis in US women
}

\author{
Wenqing Li, ${ }^{1}$ Jiali Han, ${ }^{1,2,3}$ Abrar A Qureshi ${ }^{1,2}$
}

\begin{abstract}
- Additional supplementary tables are published online only. To view the files please visit the journal online (http://ard.bmj.com/content/ early/recent)

1 Department of Dermatology, Brigham and Women's Hospital and Harvard Medical School, Boston, Massachusetts, USA ${ }^{2}$ Channing Laboratory, Department of Medicine, Brigham and Women's Hospital and Harvard Medical School, Boston, Massachusetts, USA ${ }^{3}$ Department of Epidemiology, Harvard School of Public Health, Boston, Massachusetts, USA
\end{abstract}

\section{Correspondence to}

Abrar A Qureshi, Department of Dermatology, Harvard

Medical School, Department of Dermatology, Brigham and Women's Hospital, 45 Francis Street, 221L, Boston, MA 02115, USA; aqureshi@ bics.bwh.harvard.edu

Accepted 8 April 2012

\begin{abstract}
Objectives Both overall and central obesity have been associated with the risk of psoriasis in a prospective study. Data on the association between obesity and psoriatic arthritis (PsA) have been sparse and no evidence on obesity measures and the risk of incident PsA is available now. This study aimed to evaluate the association between obesity and the risk of incident PsA in a large cohort of women.
\end{abstract}

Methods 89049 participants were included from the Nurses Health Study II over a 14-year period (1991-2005). Information on body mass index (BMI), weight change and measures of central obesity (waist circumference, hip circumference and waist-hip ratio) was collected during the follow-up. The incidence of clinician-diagnosed PsA was ascertained and confirmed by supplementary questionnaires.

Results 146 incident PsA cases were identified during 1231693 person-years of follow-up. Among all participants, BMI was monotonically associated with an increased risk of incident PsA. Compared with BMI less than 25.0, the RR was 1.83 for BMI 25.0-29.9 (95\% Cl 1.15 to 2.89), 3.12 for BMI 30.0-34.9 (95\% Cl 1.90 to 5.11$)$ and 6.46 for $\mathrm{BMI}$ over $35.0(95 \% \mathrm{Cl} 4.11$ to 10.16). There was a graded positive association between weight change from age 18 years, measures of central obesity and risk of PsA (p for trend $<0.001$ ). The analysis among participants developing psoriasis during follow-up revealed a similar association ( $p$ for trend $<0.01$ ), indicating an increased risk of PsA associated with obesity among patients with psoriasis.

Conclusion This study provides further evidence linking obesity with the risk of incident PSA among US women.

Psoriatic arthritis (PsA) is a well-recognised comorbidity of psoriasis and an inflammatory musculoskeletal condition occurring in $0.25 \%$ of the US population. ${ }^{12}$ Previous reports indicate that PsA leads to impaired quality of life due to joint damage and deformity, as well as an increased mortality. ${ }^{1}$ Despite this major impact, there is little understanding of PsA risk factors, which limits prevention and early detection efforts.

A link between obesity and psoriasis and arthritis has been reported. Increased adiposity and weight gain have been associated with the risk of psoriasis in a prospective study. ${ }^{3}$ Obesity is a significant risk factor for osteoarthritis at sites throughout the body, especially the knee. ${ }^{4}$ The reports on obesity and rheumatoid arthritis (RA) have been conflicting, and one study only observed an increased risk of anticyclic citrullinated peptide-negative RA associated with obesity. ${ }^{6-8}$ Data on obesity and PsA are sparse and no prospective studies are available. ${ }^{9} 10$ One study observed a higher body mass index (BMI) and waist-hip ratio (WHR) between PsA and healthy controls. ${ }^{9}$ The other reported $\mathrm{BMI}$ at age 18 years as a risk factor for PsA compared with individuals with psoriasis. ${ }^{10}$ The study did not indicate current BMI as a risk factor. No data on BMI, central obesity, weight change and the risk of PsA in a prospective design are available, although we have reported an association between visceral obesity and weight change correlated with psoriasis. ${ }^{3}$ Given the differential cutaneous phenotypes between psoriasis and PsA, as well as the conflicting evidence on obesity and arthritis, the association between adiposity measurements and the risk of PsA deserves further examination in a prospective setting.

In this study, we prospectively investigated the association between BMI (BMI updated biennially during the follow-up, and BMI at age 18 years), weight change, waist circumference, hip circumference, WHR and the incidence of PsA both among all participants and among women with psoriasis from the Nurses' Health Study II (NHS II).

\section{METHODS}

\section{Study cohort}

NHS II is an ongoing longitudinal cohort of women established in 1989 when 116430 female nurses aged 25-42 years responded to a mailed questionnaire enquiring about their medical history and lifestyle practices. Biennially, updated information on lifestyle factors and medical history was collected by mailed questionnaires. The follow-up rate exceeds $90 \%$.

\section{Assessment of main exposure}

In 1989, participants responded to questions on their height, weight and weight at the age of 18 years. Weight was further recorded biennially thereafter. BMI was calculated as weight in kilograms divided by the square of height in meters $\left(\mathrm{kg} / \mathrm{m}^{2}\right)$. Weight change since age 18 years was obtained by deducting the weight at age 18 years from the current weight. We asked participants to measure waist (measured at the umbilicus) and hip circumference (measured at the largest circumference) to the nearest quarter of an inch in 1993. The validation of self-reported anthropometric measurements was evaluated among 140 NHS participants. The Pearson correlation coefficient between self-report and the average of the two technician 
measurements was 0.98 for weight, 0.91 for waist circumference and 0.87 for hip circumference. ${ }^{11}$

\section{Assessment of main outcome (PsA)}

In 2005, we asked participants about physician-diagnosed psoriasis and the diagnosis date. Of the 97476 responders, 2529 reported ever being diagnosed with psoriasis; of these, 1151 self-reported psoriasis patients diagnosis occurred after 1991. Psoriasis self-reports were confirmed by using the psoriasis screening tool, which has $99 \%$ sensitivity and $94 \%$ specificity. ${ }^{12}$ This questionnaire was mailed to 1886 participants who self-reported psoriasis and responded to the 2007 main questionnaire. A diagnosis was validated if adhering to the scoring algorithms based on multiple a priori hypotheses. One thousand six hundred and thirty-seven (87\%) responded and 1511 (92\%) were confirmed.

We confirmed the diagnosis of PsA by using the psoriatic arthritis screening and evaluation (PASE) questionnaire, which includes both symptom and function scales. ${ }^{13} 14$ A total score of 47 or greater has been shown to identify PsA with high accuracy. ${ }^{13} 14$ We observed a positive association between PASE score and the 28-joint disease activity score (E. Soriano, personal communication, 2012). PASE also has good test-retest precision and is sensitive to change to therapy. ${ }^{13}$ Furthermore, PASE can distinguish between the symptoms of PsA and osteoarthritis. ${ }^{14}$

\section{Assessment of covariates}

Biennially from 1989, the smoking status and intensity among current smokers was assessed. Data on alcohol intake were available every 4 years from 1991. Physical activity was asked in 1991, 1997, 2001 and 2005, and a good validity and reproducibility was found. ${ }^{15}$ Depressive symptoms were assessed with the mental health index 5 in 1993, 1997 and 2001, which has been shown to be valid for major depression. ${ }^{16}$ Participants reported regular antidepressant medication use biennially from 1993. Menopausal status and postmenopausal hormone use, personal history of cancer, diabetes, cardiovascular disease, hypertension and hypercholesterolaemia were collected biennially.

\section{Statistical analysis}

Two sets of analyses were performed to evaluate the risk of PsA associated with obesity among all participants, as well as among those with psoriasis. For all the main analyses, of the 97476 responders, we excluded participants who did not respond to the psoriasis question in 2005 ( $N=58)$, prevalent psoriasis or PsA in 1991 ( $\mathrm{N}=1376)$, those unable to pass the confirmation of selfreports $(\mathrm{N}=97)$ or without response to the psoriasis screening tool or PASE questionnaire ( $\mathrm{N}=467)$, PsA with missing diagnosis date $(\mathrm{N}=2)$, and participants with BMI less than 10 or missing $(\mathrm{N}=6334)$. For the analysis among total participants, we excluded incident individuals with psoriasis without musculoskeletal phenotypes in the previous follow-up period ( $N=93$ ); therefore 89049 participants remained. For the analysis among individuals with psoriasis, we excluded participants who did not develop psoriasis $(\mathrm{N}=88586), 556$ with confirmed psoriasis remained. Because we excluded self-reported psoriasis cases that were not validated in the main analysis, we also performed a sensitivity analysis by using all self-reports.

We calculated person-years from the return date of the 1991 questionnaire to the psoriasis diagnosis date, or the end of follow-up (June 2005), whichever came first. Information on BMI was categorised as less than $25.0,25.0-29.9,30.0-34.9$, or 35.0 or greater, and updated during the follow-up. The cutoffs were consistent with the classification of WHO on overweight, obesity class I and obesity class II. Given the distribution of subjects, $\mathrm{BMI}$ at age 18 years was classified as less than 21.0, 21.0-22.9, $23.0-24.9$, 25.0-29.9, or 30.0 or greater. Weight change was classified as four categories (loss or increase of <20.0, 20.0-49.9, 50.0-99.9, or $\geq 100.0 \mathrm{lbs}$ ). We analysed waist circumference, hip circumference and WHR in tertiles. Cox proportional hazards analysis stratified by age and follow-up interval was performed to calculate the age and multivariate-adjusted RR and 95\% CI. Multivariate models were adjusted for age (continuous), smoking (never, past, current with 1-14, 15-24, or $\geq 25$ cigarettes/day), vigorous physical activity (metabolic equivalent hours/week, in quintiles) and alcohol intake (0, <4.9, 5.0-9.9, 10-14.9, 15-29.9, or $\geq 30.0 \mathrm{~g} /$ day). Linear trend tests were conducted by using the median in different categories. To evaluate the change of association, we performed association analyses between obesity and the risk of PsA by including cases with PASE scores less than the first, second, third and fourth quartile, respectively.

As a sensitivity analysis, level of depressive symptoms (mental health index 5 scores, 86-100, 76-85, 53-75 or 0-52) or antidepressant medication use (never, past or current), postmenopausal hormone use (premenopausal, never or ever users), personal history of chronic diseases (yes or no, including cancer, diabetes, cardiovascular disease, hypertension and hypercholesterolaemia) were concomitantly adjusted for. Another sensitivity analysis was performed in a case-control design to include all PsA and confirmed individuals with psoriasis before and after 1991. Analyses were carried out by using SAS software (version 9.2). All $p$ values were two-tailed with the significance level set at $p<0.05$. The study was approved by the institutional review board of Partners Health Care System. The participants' return of a completed questionnaire was accepted as informed consent to the present study.

\section{RESULTS}

Participants with higher BMI were more likely to be older and tended to have less alcohol intake and less physical activity. We observed an increase in weight at age 18 years, weight gain, waist and hip circumference and WHR, with increasing categories of BMI in 1991 (table 1).

We documented 146 incident PsA cases during 1231693 person-years of follow-up. The risk of PsA was monotonically elevated with increasing BMI ( $p$ for trend $<0.0001$ ). Compared with BMI less than 25.0, the risk of BMI 25.0-29.9, 30.0-34.9 and 35.0 or greater increased to 1.83 (95\% CI 1.15 to 2.89 ), 3.12 (95\% CI 1.90 to 5.11 ) and 6.46 (95\% CI 4.11 to 10.16$)$, respectively (table 2). There was a trend towards an increased risk of PsA by weight change since the age of 18 years and the RR of PsA by weight gain of $10 \mathrm{lb}$ was 1.17 (95\% CI 1.12 to 1.22). The positively graded association persisted when analysing the central adiposity measures ( $p$ for trend <0.001). When one measure of central obesity (waist circumference, hip circumference, or WHR) was included in the model with BMI, its association remained significant except for WHR after adjusting for BMI ( $p$ for trend=0.064) (table 2 and supplementary tables S1 and S2, available online only).

We repeated all the analyses by excluding participants without developing psoriasis to examine the risk of PsA among individuals with psoriasis (table 3). Participants with BMI of 35.0 or greater were 2.98 times more likely to develop PsA (95\% CI 1.86 to $4.78, \mathrm{p}$ for trend $<0.0001$ ). Similarly, the risk of PsA among confirmed psoriasis was monotonically elevated with weight change ( $p$ for trend $<0.0001$ ) and measures of central obesity ( $p$ for trend all <0.01). We also evaluated the effect estimates 
Table 1 Characteristics of study participants by body mass index in 1991, Nurses' Health Study II*

\begin{tabular}{|c|c|c|c|c|}
\hline & \multicolumn{4}{|c|}{ Body mass index } \\
\hline $\mathrm{n}$ & 58947 & 18225 & 7144 & 4733 \\
\hline Current smokers (yes, \%) & 11.3 & 12.1 & 11.4 & 10.9 \\
\hline Alcohol intake, g/day, mean (SD) & $3.5(6.3)$ & $2.8(6.0)$ & $2.0(5.0)$ & $1.6(4.7)$ \\
\hline Vigorous physical activity, metabolic equivalent hours/week, mean (SD) & $15.3(24.3)$ & $11.6(19.2)$ & $9.4(16.1)$ & $7.5(14.2)$ \\
\hline Weight change from age 18 years, lb, mean (SD) & $9.3(13.4)$ & $28.8(18.4)$ & $47.9(23.1)$ & $75.9(33.9)$ \\
\hline Waist circumference, inches, mean (SD) & $28.8(3.1)$ & $33.5(3.9)$ & $37.9(4.5)$ & $42.8(5.7)$ \\
\hline Hip circumference, inches, mean (SD) & $37.5(2.6)$ & $41.7(3.3)$ & $45.6(3.9)$ & $51.0(5.4)$ \\
\hline Waist-hip ratio, mean (SD) & $0.77(0.07)$ & $0.81(0.08)$ & $0.83(0.08)$ & $0.84(0.09)$ \\
\hline Mental health index $\leq 52$ (yes, \%) & 12.2 & 14.1 & 15.9 & 17.4 \\
\hline Antidepressant use $($ yes, \%) $\ddagger$ & 9.6 & 11.5 & 13.5 & 17.1 \\
\hline Cardiovascular disease & 0.4 & 0.4 & 0.7 & 0.9 \\
\hline Hypertension & 1.6 & 4.0 & 8.0 & 14.7 \\
\hline Hypercholesterolaemia & 7.3 & 11.7 & 16.3 & 16.9 \\
\hline
\end{tabular}

${ }^{*}$ Characteristics of participants at the beginning of follow-up (return date of the 1991 questionnaire). Values are means (SD) or percentages and are standardised to the age distribution of the study population.

†Values are not age-adjusted.

¥Mental health index and antidepressant use in 1993 .

by concomitantly adjusting for BMI and one measure of central obesity, BMI and WHR remained significant when cross-adjusting (table 3 and supplementary tables S1 and S2, available online only). The effect values of BMI remained similar across analyses adjusting for measures of central obesity.

We compared the association between obesity and PsA with severity scores less than the first (49), second (53), third (58) and fourth (75) quartile both among the total participants and among individuals with psoriasis. We observed an elevation of RR when including PsA with the higher PASE scores (data not shown).

Stratified analysis by smoking or physical activity did not find any material differences. We performed secondary analyses by adjusting for the level of depressive symptomatology or antidepressive medication use, postmenopausal hormone use and personal history of chronic diseases and no material change of the results was observed. Sensitivity analysis was carried out to examine the association between obesity and PsA among all self-reported individuals with psoriasis (data not shown). A case-control analysis was conducted to incorporate the information of prevalent cases (supplementary tables S3 and S4, available online only) and the association remains robust.

\section{DISCUSSION}

We prospectively evaluated the association between measures of adiposity and the risk of incident PsA in a well-established cohort of women. Our results indicated a markedly accumulated risk of PsA, correlated with BMI, weight change since early adulthood, waist and hip circumference and WHR, both among total participants and among women with psoriasis. These associations existed in a dose-dependent fashion, highlighting the effect of adiposity in the development of PsA.

The cutaneous and musculoskeletal effects of adiposity have received great interest recently. Obesity was demonstrated as an independent risk factor for psoriasis in our prospective study. ${ }^{3}$
A cross-sectional study also pointed to the link particularly among those with severe psoriasis. ${ }^{17}$ However, there is still a lack of agreement on the severity of skin phenotypes between psoriasis and PsA. 1819 The adverse effect of obesity on the risk of osteoarthritis has been published. ${ }^{4}$ Although obesity was found to be associated with an increased risk of RA, several recent studies did not support this association, as reviewed by Stavropoulos-Kalinoglou et al. ${ }^{6}$ Interestingly, RA patients with high BMI have lower mortality than thinner patients. ${ }^{20}$ Given the marked differences between PsA and other types of arthritis, addressing the role of obesity in PsA development was needed. Moreover, in this study, we were able to evaluate the correlation between obesity and PsA both among the total participants and among those with psoriasis.

In general, there are sparse data on the association between obesity and PsA. Tam et al observed a higher current BMI and WHR among PsA compared with healthy controls, but failed to find a significant difference for waist circumference. SoltaniArabshahi et al ${ }^{10}$ suggested that BMI in early adulthood increased the risk of PsA, but they did not observe an association between current BMI or other measures of obesity and PsA. In addition, the study design left uncertainty regarding the temporal relationship and did not allow for causal inference. In our prospective analysis, BMI conveys a significantly increased risk of PsA in a dose-dependent manner. In contrary to a report on RA, ${ }^{20}$ the association was stronger in our study, when including more severe PsA, as defined by higher PASE scores. Weight gain from early adulthood and measures of central obesity also monotonically increased the risk. The effect was robust among never and ever smokers, those with more or less physical activity, comprehensively demonstrating the independent role of high adiposity in the development of PsA.

Obesity at age 18 years appears to be the only measure that became null when we performed the analyses among psoriasis cases, which did not replicate the observation among total 
Table 2 Age and multivariate-adjusted RR for the association of adiposity measurements with risk of psoriatic arthritis among all participants*

\begin{tabular}{|c|c|c|c|c|}
\hline & Cases & Person-years & $\begin{array}{l}\text { Age-adjusted RR } \\
(95 \% \mathrm{Cl})\end{array}$ & $\begin{array}{l}\text { Multivariate-adjusted } \\
\text { RRt }(95 \% \mathrm{Cl})\end{array}$ \\
\hline Updated BMI $\left(\mathrm{kg} / \mathrm{m}^{2}\right)$ & 146 & 1231693 & & \\
\hline$<25.0$ & 40 & 703190 & 1.00 & 1.00 \\
\hline $25.0-29.9$ & 35 & 297149 & 1.88 (1.19 to 2.96$)$ & 1.83 (1.15 to 2.89$)$ \\
\hline $30.0-34.9$ & 28 & 133146 & $3.22(1.98$ to 5.26$)$ & $3.12(1.90$ to 5.11$)$ \\
\hline$\geq 35.0$ & 43 & 98208 & 6.60 (4.26 to 10.23$)$ & 6.46 (4.11 to 10.16$)$ \\
\hline $\mathrm{p}$ for trend & & & $<0.0001$ & $<0.0001$ \\
\hline $\mathrm{BMI}$ at age 18 years $\left(\mathrm{kg} / \mathrm{m}^{2}\right)$ & 145 & 1222076 & & \\
\hline$<21.0$ & 67 & 701559 & 1.00 (0.64 to 1.58$)$ & 1.04 (0.66 to 1.64$)$ \\
\hline $21.0-22.9$ & 26 & 272700 & 1.00 & 1.00 \\
\hline $23.0-24.9$ & 24 & 125453 & 2.01 (1.15 to 3.50$)$ & 1.93 (1.11 to 3.37$)$ \\
\hline $25.0-29.9$ & 17 & 93857 & $1.88(1.02$ to 3.46$)$ & $1.74(0.94$ to 3.21$)$ \\
\hline$\geq 30.0$ & 11 & 28508 & 4.07 (2.01 to 8.24$)$ & 3.55 (1.75 to 7.23$)$ \\
\hline $\mathrm{p}$ for trend & & & $<0.0001$ & $<0.0001$ \\
\hline Weight change from age 18 years (lb) & 145 & 1222076 & & \\
\hline Loss or increase of $<20.0$ & 32 & 563364 & 1.00 & 1.00 \\
\hline Increase of 20.0-49.9 & 45 & 435087 & 1.68 (1.06 to 2.65$)$ & 1.72 (1.09 to 2.72$)$ \\
\hline Increase of 50.0-99.9 & 50 & 191981 & $3.88(2.46$ to 6.13$)$ & 3.67 (2.31 to 5.84$)$ \\
\hline Increase of $\geq 100.0$ & 18 & 31645 & 8.09 (4.48 to 14.64$)$ & 7.00 (3.78 to 12.96$)$ \\
\hline $\mathrm{p}$ for trend & & & $<0.0001$ & $<0.0001$ \\
\hline Waist circumference (inches) & 72 & 599200 & & \\
\hline Tertile $1,<28.0$ & 7 & 168183 & 1.00 & 1.00 \\
\hline Tertile 2, 28.0-31.9 & 17 & 227235 & $1.72(0.71$ to 4.16$)$ & $1.65(0.68$ to 4.00$)$ \\
\hline Tertile $3, \geq 32.0$ & 48 & 203782 & 5.05 (2.28 to 11.20$)$ & 4.82 (2.15 to 10.83$)$ \\
\hline $\mathrm{p}$ for trend & & & $<0.0001$ & $<0.0001$ \\
\hline Hip circumference (inches) & 70 & 597544 & & \\
\hline Tertile $1,<38.0$ & 13 & 233613 & 1.00 & 1.00 \\
\hline Tertile 2, 38.0-40.9 & 13 & 187362 & $1.18(0.55$ to 2.56$)$ & 1.25 (0.58 to 2.72$)$ \\
\hline Tertile $3, \geq 41.0$ & 44 & 176569 & 4.02 (2.16 to 7.51$)$ & 4.32 (2.27 to 8.24$)$ \\
\hline$p$ for trend & & & $<0.0001$ & $<0.0001$ \\
\hline Waist-hip ratio & 70 & 596835 & & \\
\hline Tertile $1,<0.744$ & 12 & 199028 & 1.00 & 1.00 \\
\hline Tertile $2,0.744-0.800$ & 20 & 207096 & $1.58(0.77$ to 3.23$)$ & $1.49(0.73$ to 3.06$)$ \\
\hline Tertile $3,>0.800$ & 38 & 190712 & 3.12 (1.63 to 5.98$)$ & 2.84 (1.48 to 5.48$)$ \\
\hline$p$ for trend & & & 0.0002 & 0.0006 \\
\hline
\end{tabular}

*Psoriasis cases with only skin phenotypes were excluded during the follow-up.

†Adjusted for age (continuous variable), smoking (never, past, current with 1-14, 15-24, or $\geq 25$ cigarettes/day), alcohol drinking (no, $<4.9,5.0-9.9,10-14.9,15-29.9$ or $\geq 30.0 \mathrm{~g} /$ day), vigorous physical activity (metabolic equivalent hours/week, in quintile), and height (inches, for the analysis of waist circumference, hip circumference and waist-hip ratio), and weight at 18 years (for the analysis of weight change from 18 years).

BMI, body mass index.

participants and of a previous report. ${ }^{10}$ On the other hand, weight gain from age 18 years was robustly associated with the risk of PsA with a beyond $10 \%$ elevated risk per $10 \mathrm{lb}$ weight increase. Weight change rather than weight at early adulthood may serve as a major relevant factor.

Anthropometric measures of abdominal obesity have been proposed as substantial indicators of health risk. ${ }^{21-23}$ Our analysis showed similar trends in the risk of PsA associated with waist circumference, hip circumference and WHR. Cross-adjustment of BMI and measures of central obesity reached similar effect values of BMI, even some of which did not fulfil statistical significance possibly due to the increased standard error by co-linearity. Central obesity measures were greatly attenuated but still had a residual effect on the risk of PsA even after adjusting for BMI. Stratified analysis by BMI indicated especially for the non-obese participants $\left(\mathrm{BMI}<30 \mathrm{~kg} / \mathrm{m}^{2}\right)$ central obesity was significantly associated with the risk of PsA.

Inflammation may be the key mechanism underlying our findings. Obesity in psoriasis has been associated with both decreased plasma levels of adiponectin and enhanced systemic inflammation and oxidative stress. ${ }^{24-28}$ Adiposity can augment cytokine expression by the recruited inflammatory infiltrate, such as interleukin 6 and tumour necrosis factor $\alpha$, relevant to psoriasis pathophysiology. ${ }^{26-28}$ The leptin and resistin overload may elicit the cutaneous pro-inflammatory changes in psoriasis. ${ }^{29}$ Given that PsA is a well-recognised systemic inflammatory disorder, a fundamental pathological process that leads to PsA could be the chronic inflammatory state induced by adiposity. ${ }^{12}$ Alternatively, obesity and PsA may share some still unknown common cause. Despite a statistical association, it is early to jump to a conclusion about a causal link. Obesity may serve as a surrogate endpoint for other risk factors of PsA, although smoking or physical activity does not seem to modify the association. Another possible mechanism to explain this link is mental health disorders. Psoriasis has been associated with depression, which appears to be reciprocally correlated with obesity. ${ }^{30} 31$ We observed a higher percentage of participants with depression symptoms and antidepressant medication use among the obese. However, sensitivity analyses accounting for depression did not materially change the results in this study, indicating that the role of obesity is independent of depression. 
Table 3 Age and multivariate-adjusted RRs for the association of adiposity measurements with risk of psoriatic arthritis among participants with confirmed psoriasis

\begin{tabular}{|c|c|c|c|c|}
\hline & Cases & Person-years & $\begin{array}{l}\text { Age-adjusted RR } \\
(95 \% \text { Cl) }\end{array}$ & $\begin{array}{l}\text { Multivariate-adjusted } \\
\left.\text { RR* }^{*} 95 \% \mathrm{Cl}\right)\end{array}$ \\
\hline Updated BMI (kg/m²) & 146 & 6838 & & \\
\hline$<25.0$ & 40 & 3245 & 1.00 & 1.00 \\
\hline $25.0-29.9$ & 35 & 1533 & 1.80 (1.12 to 2.88$)$ & 1.81 (1.12 to 2.93$)$ \\
\hline $30.0-34.9$ & 28 & 1051 & 1.98 (1.19 to 3.28$)$ & $1.90(1.13$ to 3.18$)$ \\
\hline$\geq 35.0$ & 43 & 1009 & 2.97 (1.88 to 4.69$)$ & 2.98 (1.86 to 4.78$)$ \\
\hline $\mathrm{p}$ for trend & & & $<0.0001$ & $<0.0001$ \\
\hline $\mathrm{BMI}$ at age 18 years $\left(\mathrm{kg} / \mathrm{m}^{2}\right)$ & 145 & 6791 & & \\
\hline$<21.0$ & 67 & 3265 & $1.23(0.77$ to 1.96$)$ & 1.28 (0.79 to 2.06$)$ \\
\hline $21.0-22.9$ & 26 & 1668 & 1.00 & 1.00 \\
\hline $23.0-24.9$ & 24 & 858 & $1.74(0.98$ to 3.09$)$ & $1.73(0.96$ to 3.13$)$ \\
\hline $25.0-29.9$ & 17 & 610 & $1.69(0.89$ to 3.20$)$ & $1.69(0.88$ to 3.26$)$ \\
\hline$\geq 30.0$ & 11 & 390 & 1.61 (0.76 to 3.42$)$ & 1.53 (0.71 to 3.29$)$ \\
\hline $\mathrm{p}$ for trend & & & 0.10 & 0.20 \\
\hline Weight change from age 18 years (lb) & 145 & 6791 & & \\
\hline Loss or increase of $<20.0$ & 32 & 2669 & 1.00 & 1.00 \\
\hline Increase of $20.0-49.9$ & 45 & 2285 & $1.36(0.84$ to 2.18$)$ & 1.34 (0.82 to 2.17$)$ \\
\hline Increase of 50.0-99.9 & 50 & 1513 & 2.31 (1.46 to 3.68$)$ & 2.42 (1.49 to 3.91$)$ \\
\hline Increase of $\geq 100.0$ & 18 & 324 & 3.28 (1.76 to 6.11$)$ & 3.84 (1.93 to 7.63$)$ \\
\hline $\mathrm{p}$ for trend & & & $<0.0001$ & $<0.0001$ \\
\hline Waist circumference (inches) & 72 & 3272 & & \\
\hline Tertile $1,<28.0$ & 7 & 746 & 1.00 & 1.00 \\
\hline Tertile 2, 28.0-31.9 & 17 & 1045 & $1.67(0.63$ to 4.38$)$ & $1.46(0.54$ to 3.99$)$ \\
\hline Tertile $3, \geq 32.0$ & 48 & 1481 & $3.23(1.36$ to 7.69$)$ & $3.02(1.21$ to 7.56$)$ \\
\hline $\mathrm{p}$ for trend & & & 0.002 & 0.004 \\
\hline Hip circumference (inches) & 70 & 3245 & & \\
\hline Tertile $1,<38.0$ & 13 & 931 & 1.00 & 1.00 \\
\hline Tertile 2, 38.0-40.9 & 13 & 917 & $1.31(0.56$ to 3.05$)$ & 1.24 (0.51 to 3.00$)$ \\
\hline Tertile $3, \geq 41.0$ & 44 & 1397 & 2.35 (1.15 to 4.79$)$ & 2.59 (1.18 to 5.69$)$ \\
\hline $\mathrm{p}$ for trend & & & 0.009 & 0.006 \\
\hline Waist-hip ratio & 70 & 3245 & & \\
\hline Tertile $1,<0.744$ & 12 & 1008 & 1.00 & 1.00 \\
\hline Tertile 2, $0.744-0.800$ & 20 & 996 & $1.43(0.67$ to 3.06$)$ & $1.41(0.63$ to 3.15$)$ \\
\hline Tertile $3,>0.800$ & 38 & 1241 & 2.36 (1.18 to 4.74$)$ & $2.48(1.20$ to 5.15$)$ \\
\hline$p$ for trend & & & 0.009 & 0.008 \\
\hline
\end{tabular}

\footnotetext{
*Adjusted for age (continuous variable), smoking (never, past, current with 1-14, 15-24, or $\geq 25$ cigarettes/day), alcohol drinking (no, $<4.9,5.0-9.9,10-14.9,15-29.9$ or $\geq 30.0 \mathrm{~g} /$ day), vigorous physical activity (metabolic equivalent hours/week, in quintile), and height (inches, for the analysis of waist circumference, hip circumference and waist-hip ratio) and weight at 18 years (for the analysis of weight change from 18 years).

BMI, body mass index.
}

To our knowledge, this is the first prospective study on this topic. We observed a compelling association by employing multiple markers of obesity to probe into the risk of PsA. Because the anthropometric measures may change over time, using updated BMI and weight change over time instead of only BMI at baseline allowed us to evaluate the harms of continuing gain in weight and also to evaluate the benefits of weight loss. Our study was reasonably powered. A variety of sensitivity analyses were applied and the results did not differ appreciably, arguing for the robustness of the observations.

Our study also has retrospective characteristics and selection and information bias may be a concern. Survivorship bias would be a major concern on the selection of participants given that the psoriasis question was asked in 2005 . However, a cohort of younger women ensures our results were less likely to be greatly distorted. The mean age of those who did not respond to the psoriasis question was even slightly younger compared with responders. Potential recall bias may be caused by retrospective enquiry of the main outcome. However, the healthcare-related professional background of our participants was reassuring and psoriasis self-reports have reached a confirmation rate of $92 \% .{ }^{12}$ Case ascertainment of clinician-diagnosed PsA by PASE questionnaire among selfreported individuals with psoriasis could be another major concern, which may lead to misclassification bias. However, PASE was testified to be a valid and reliable screening tool for PsA, particularly active PsA among psoriasis cases in previous pilot studies. ${ }^{13} 14$ The prevalence of PsA among individuals with confirmed psoriasis from 1991 is $21.2 \%$, falling within the range of previous reports. ${ }^{1}$ There may be concerns about possible misclassification with other musculoskeletal diseases. Previous studies reported an adverse effect of obesity on osteoarthritis and fibromyalgia. ${ }^{4} 52$ However, PASE can distinguish the symptoms of PsA from osteoarthritis, albeit less of a concern in a younger cohort. Fibromyalgia seldom occurs in patients with psoriasis. It would be worth noting that central obesity was only asked once during the follow-up, while we had the opportunity to update BMI and weight change over time. However, a previous study has indicated the validity of the measurements. ${ }^{11}$ PsA cases without concomitant psoriasis were excluded in our analysis. The effect of obesity on these cases, known as seronegative inflammatory arthritis or spondyloarthritis, needs to be clarified further. The participants were primarily younger and middle-aged female Caucasians. 
Although the biological effects of adiposity should be similar and the homogeneity of study participants led to less confounding by socioeconomic status, the generalisability to other populations, particularly men, and other racial/ethnic minorities, may be made cautiously.

In conclusion, our large well-characterised cohort study provides evidence of a dose-dependent relationship between overall obesity, central adiposity and an increased risk of PsA in women. The effect of obesity on PsA goes beyond that on psoriasis skin phenotypes alone. In this issue, another paper on a similar topic by Love et a ${ }^{\beta 3}$ was also published, which followed up a cohort of psoriasis patients by general practitioners in northern UK, providing further testifying evidence to our study. The implication of the observations may be substantial as obesity is a modifiable factor that is becoming increasingly prevalent. Further studies are warranted to elucidate the underlying mechanisms and clarify the causative association.

Contributors AAQ had full access to all of the data in the study and takes responsibility for the integrity of the data and the accuracy of the data analysis. Study concept and design: AAQ, JH and WL. Acquisition of data: AAO and JH. Analysis and interpretation of data: WL, AAQ and JH. Drafting of the manuscript: $\mathrm{WL}, \mathrm{AAO}$ and $\mathrm{JH}$. Critical revision of the manuscript for important intellectual content: WL, AAQ and JH. Statistical analysis: WL. Obtained funding: AAO. Administrative, technical, or material support: $A A Q$ and JH. Study supervision: $\mathrm{WL}, \mathrm{AAO}$ and $\mathrm{JH}$.

Funding The work is supported by the Department of Dermatology, Brigham and Women's Hospital, NHS II grant R01 CA50385.

Competing interests $A A Q$ has received a grant from Amgen/Pfizer to evaluate 'Biomarkers in psoriasis and psoriatic arthritis'. AAO also serves as a consultant for Abbott, Centocor, Novaritis and the Centers for Disease Control and Prevention. The other authors state no conflict of interest.

Ethics approval The institutional review btoard of Partners Health Care System approved this study.

Patient consent Obtained.

Provenance and peer review Not commissioned; externally peer reviewed.

\section{REFERENCES}

1. Gelfand JM, Gladman DD, Mease PJ, et al. Epidemiology of psoriatic arthritis in the population of the United States. J Am Acad Dermatol 2005;53:573.

2. Nograles KE, Brasington RD, Bowcock AM. New insights into the pathogenesis and genetics of psoriatic arthritis. Nat Clin Pract Rheumatol 2009;5:83-91.

3. Setty AR, Curhan G, Choi HK. Obesity, waist circumference, weight change, and the risk of psoriasis in women: Nurses' Health Study II. Arch Intern Med 2007;167:1670-5.

4. Griffin TM, Huebner JL, Kraus VB, et al. Induction of osteoarthritis and metabolic inflammation by a very high-fat diet in mice: effects of short-term exercise. Arthritis Rheum 2012;64:443-53.

5. Sowers MR, Karvonen-Gutierrez CA. The evolving role of obesity in knee osteoarthritis. Curr Opin Rheumatol 2010:22:533-7.

6. Stavropoulos-Kalinoglou A, Metsios GS, Koutedakis Y, et al. Obesity in rheumatoid arthritis. Rheumatology (Oxford) 2011:50:450-62.

7. Pattison $\mathbf{E}$, Harrison BJ, Griffiths $\mathrm{CE}$, et al. Environmental risk factors for the development of psoriatic arthritis: results from a case-control study. Ann Rheum Dis 2008;67:672-6.
8. Pedersen M, Jacobsen $\mathrm{S}$, Klarlund $\mathrm{M}$, et al. Environmental risk factors differ between rheumatoid arthritis with and without auto-antibodies against cyclic citrullinated peptides. Arthritis Res Ther 2006;8:R133.

9. Tam LS, Tomlinson B, Chu TT, et al. Cardiovascular risk profile of patients with psoriatic arthritis compared to controls-the role of inflammation. Rheumatology (Oxford) 2008:47:718-23

10. Soltani-Arabshahi R, Wong B, Feng BJ, et al. Obesity in early adulthood as a risk factor for psoriatic arthritis. Arch Dermatol 2010;146:721-6.

11. Rimm EB, Stampfer MJ, Colditz GA, et al. Validity of self-reported waist and hip circumferences in men and women. Epidemiology 1990;1:466-73.

12. Dominguez PL, Assarpour A, Kuo H, et al. Development and pilot-testing of a psoriasis screening tool. Br J Dermatol 2009:161:778-84.

13. Dominguez PL, Husni ME, Holt EW, et al. Validity, reliability, and sensitivity-tochange properties of the psoriatic arthritis screening and evaluation questionnaire. Arch Dermatol Res 2009;301:573-9.

14. Husni ME, Meyer KH, Cohen DS, et al. The PASE questionnaire: pilot-testing a psoriatic arthritis screening and evaluation tool. J Am Acad Dermatol 2007;57:581-7.

15. Wolf AM, Hunter DJ, Colditz GA, et al. Reproducibility and validity of a selfadministered physical activity questionnaire. Int J Epidemiol 1994;23:991-9.

16. Berwick DM, Murphy JM, Goldman PA, et al. Performance of a five-item mental health screening test. Med Care 1991;29:169-76.

17. Neimann AL, Shin DB, Wang $X$, et al. Prevalence of cardiovascular risk factors in patients with psoriasis. J Am Acad Dermatol 2006;55:829-35.

18. Elkayam 0, Ophir J, Yaron M, et al. Psoriatic arthritis: interrelationships between skin and joint manifestations related to onset, course and distribution. Clin Rheumatol 2000; 19:301-5.

19. Gisondi $\mathbf{P}$, Tinazzi I, Del Giglio M, et al. The diagnostic and therapeutic challenge of early psoriatic arthritis. Dermatology (Basel) 2010;221 (Suppl 1):6-14.

20. Escalante A, Haas RW, del Rincón I. Paradoxical effect of body mass index on survival in rheumatoid arthritis: role of comorbidity and systemic inflammation. Arch Intern Med 2005;165:1624-9.

21. Bigaard J, Thomsen BL, Tjønneland A, et al. Does waist circumference alone explain obesity-related health risk? Am J Clin Nutr 2004;80:790-1; author reply 791-2.

22. Bray GA. Don't throw the baby out with the bath water. Am J Clin Nutr 2004; 79:347-9.

23. Janssen I, Katzmarzyk PT, Ross R. Waist circumference and not body mass index explains obesity-related health risk. Am J Clin Nutr 2004;79:379-84.

24. Gerdes S, Rostami-Yazdi M, Mrowietz U. Adipokines and psoriasis. Exp Dermatol 2011:20:81-7.

25. Kaur S, Zilmer K, Kairane C, et al. Clear differences in adiponectin level and glutathione redox status revealed in obese and normal-weight patients with psoriasis. Br J Dermatol 2008;159:1364-7.

26. Davidovici BB, Sattar N, Prinz JC, et al. Psoriasis and systemic inflammatory diseases: potential mechanistic links between skin disease and co-morbid conditions. $J$ Invest Dermatol 2010;130:1785-96.

27. Hamminga EA, van der Lely AJ, Neumann HA, et al. Chronic inflammation in psoriasis and obesity: implications for therapy. Med Hypotheses 2006;67:768-73.

28. Sterry W, Strober BE, Menter A. Obesity in psoriasis: the metabolic, clinical and therapeutic implications. Report of an interdisciplinary conference and review. Br J Dermatol 2007:157:649-55.

29. Johnston A, Arnadottir S, Gudjonsson JE, et al. Obesity in psoriasis: leptin and resistin as mediators of cutaneous inflammation. Br J Dermatol 2008;159: $342-50$.

30. Luppino FS, de Wit LM, Bouvy PF, et al. Overweight, obesity, and depression: a systematic review and meta-analysis of longitudinal studies. Arch Gen Psychiatry 2010;67:220-9.

31. Campolmi E, Zanieri F, Santosuosso U, et al. The importance of stressful family events in psoriatic patients: a retrospective study. J Eur Acad Dermatol Venereol [Epub ahead of print 29 Sep 2011]. doi: 10.1111/j.1468-3083.2011.04268.x.

32. Ursini F, Naty S, Grembiale RD. Fibromyalgia and obesity: the hidden link. Rheumatol Int 2011;31:1403-8.

33. Love TJ, Zhu Y, Zhang Y, et al. Obesity and the risk of psoriatic arthritis: a population-based study. Ann Rheum Dis 2012;11:1273-7. 